\title{
Peak Load Cutting in District Heating Network
}

\author{
Petri Hietaharju Mika Ruusunen \\ Control Engineering, University of Oulu, Finland, \{petri.hietaharju, mika.ruusunen\} @oulu.fi
}

\begin{abstract}
Simulations of different peak load cutting scenarios in district heating of buildings were performed. Decrease in percentages of $30 \%, 50 \%$, and $70 \%$ in peak loads was analyzed for the two modelled apartment buildings. Simulation results show that even $70 \%$ peak load cuts are possible in individual buildings. However, results also reveal that for some buildings 30\% peak load cuts would require compromising with the indoor temperature. Therefore, it is important to take into account the different heat storing capacities available in each of the buildings. In future, systems with multiple buildings will be studied to effectively utilize individual heat storing capacities to cut city level peak loads. Simulations presented in this article show that better energy efficiency in district heating can be achieved by predicting the energy consumption and utilizing the thermal mass of a building.
\end{abstract}

Keywords: district heating, peak load cutting, optimization, indoor temperature prediction, modelling

\section{Introduction}

Heat represents more than half of the world's total energy consumption and three-quarters of the fuels used to meet this heat demand consist of fossil fuels (Eisentraut and Brown, 2014). Despite these facts, heat is largely ignored in the climate change debate. Nevertheless, it is important to implement new energy efficiency measures in the heat sector. Cutting peak loads in district heating network is one of such measures and in this work peak load cutting is studied with simulations.

Peak loads in district heating network occur when the heat demand exceeds the production capacity of available heating power. This means that reserve power plants need to be started to satisfy the heating demand. This raises production costs (and also environmental impact) for the energy producer as more expensive oil is used for fuel instead of wood, peat or coal. Therefore, it is in the interest of energy companies to cut peak loads and reduce the use of oil. Additionally, $\mathrm{CO}_{2}$ emissions are also reduced. At the same time, more accurate and stable indoor temperature control could be achieved by implementing the optimization routines for energy consumption.
A concept for peak load cutting has been presented in (Hietaharju and Ruusunen, 2015). The concept aims to cut peak loads by utilizing building thermal mass as a short term heat storage. Building thermal mass and its use in peak load cutting has also been discussed in (Braun, 2013; Henze et al., 2007; Sun et al., 2013; Kensby et al., 2015; Hagentoft and Kalagasidis, 2015; Ståhl, 2009). Braun (2013) presented a review on load control utilizing building thermal mass including simulation, laboratory and field studies. It showed that there is significant saving potential for using building thermal mass, but it is affected by many factors, including utility rates, type of equipment, occupancy schedule, building construction, climate conditions, and control strategy. These factors were further studied by Henze et al. (2007) using a sensitivity analysis. Sun et al. (2013) presented a more recent look into peak load cutting. They found that in existing studies more than $30 \%$ daily peak load reduction and also significant overall cost savings from $8.5 \%$ to $29 \%$ had been achieved utilizing building thermal mass. They also found that there exists model based as well as model free solutions. The amount of energy stored in the building thermal mass is difficult to identify and model based solutions are needed, but they mention the difficulties related to complex physical models and their identification.

Kensby et al. (2015) demonstrated that heavy buildings can tolerate relatively large variations in district heating energy while still maintaining desired indoor temperature. The effect of heating power reduction on indoor temperature was also studied by Hagentoft and Kalagasidis (2015). In case of $1{ }^{\circ} \mathrm{C}$ change in the control signal, the indoor temperature drop was below $0.2{ }^{\circ} \mathrm{C}$ after 24 hours, which shows that the building thermal mass can be potentially utilized for peak load cutting. However, they mention that the results are highly dependent on the thermal characteristics of the building. In that regard, Ståhl (2009) found that thermal effusivity, which is a function of thermal conductivity and heat capacity and represents the materials ability to exchange thermal energy with its surroundings, is the most important parameter when considering the heat storage capacity of a building. Heavy buildings have higher thermal effusivity and therefore offer higher energy storage capacity compared with light buildings with lower thermal effusivity. Also, 
the indoor temperature is typically more stable in heavy buildings.

To utilize thermal mass effectively to cut peak loads and optimize heat consumption, one has to be able to predict the future heat demand. This can be achieved by modelling the thermal behavior of a building, namely the indoor temperature. This way also the quality of living for the residents can be ensured by maintaining the indoor temperature at acceptable level despite the cuts in heating power. In this work, an indoor temperature model (Hietaharju et al., unpublished) was applied to simulate peak load cutting in two apartment buildings. Finally, simulation results for different peak load cutting scenarios are presented and discussed.

\section{Data}

Two apartment buildings located in the city of Jyväskylä in Finland were studied. Building 1 was constructed in 2011 whereas Building 2 was constructed in 1972 and renovated in 1993. Basic information about the buildings is presented in Table 1. Ground plans and elevation drawings were also available for the studied buildings.

Measurement data for both of the buildings was acquired in early January 2015. Data included hourly values for heating power. In addition, outdoor temperature measurements were recorded for the same time period. Hourly indoor temperature measurements for both buildings were also available. Both buildings contained several indoor temperature measurements which were located in living rooms and hallways.

Table 1. Building Information.

\begin{tabular}{lll}
\hline & Building 1 & Building 2 \\
\hline Year of construction & 2011 & 1972 \\
Year of renovation & - & 1993 \\
Floors & 4 & 7 \\
Apartments & 75 & 53 \\
Living space $\left(\mathrm{m}^{2}\right)$ & 3563 & 3024 \\
Floor space $\left(\mathrm{m}^{2}\right)$ & 4200 & 3703 \\
Volume $\left(\mathrm{m}^{3}\right)$ & 15617 & 12400 \\
\hline
\end{tabular}

\section{Methods}

Previously identified and validated (Hietaharju et al., unpublished) indoor temperature model was used for predicting the indoor temperature evolution over time in the buildings. The model structure (Equation 1) is based on Newton's cooling law and includes heat capacity $(C)$ and heat loss coefficient $(U)$ as physical parameters. Inputs for the model are indoor temperature $\left(T_{i}\right)$, outdoor temperature $\left(T_{o}\right)$, and heating power $(P)$ which can include a lag of $k$ hours. Time step $(\Delta t)$ for the model is one hour. Model output is the hourly indoor temperature along the defined prediction horizon.

$$
\begin{aligned}
T_{i, t}= & a\left(T_{i, t-1}+\frac{\Delta t}{C}\left(P_{t-1}\right.\right. \\
& \left.\left.-U\left(T_{i, t-1}-T_{o, t-1}\right)\right)\right)+b
\end{aligned}
$$

Initial data described in Section 2, including ground plans and elevation drawings, was used to calculate physical model parameters. Some assumptions were made about the construction materials due to insufficient information. After the calculation of the physical model parameters, input data mentioned before was used to estimate additional model parameters $a$ and $b$. The indoor temperature model was then utilized to optimize heating power in pilot buildings considering different peak load cutting scenarios.

The scenarios for cutting the peak loads considered simulations for $30 \%, 50 \%$, and $70 \%$ reduction in the heating power. These cuts were made at the morning hours between 7 and $10 \mathrm{am}$. Load cuts were calculated from the actual measured district heating power. During the simulations, maximum allowed power was restricted accordingly during the peak load hours. Cost function for peak load cutting minimized the power consumption while keeping the indoor temperature between the control limits. This was achieved with a constraint by increasing the cost function value if the indoor temperature would have exceeded the limits according to the model prediction. Also the increase and decrease in the amount of heating power was restricted to prevent too large hourly power changes.

All modelling and optimization work was programmed and evaluated in MATLAB ${ }^{\circledR}$ software with simulations. MATLAB ${ }^{\circledR}$ 's simulated annealing algorithm was utilized in peak load cutting simulations to optimize the usage of heat energy per building with respect to the constraint. In all the succeeding simulations, the prediction horizon of 100 hours was applied with acquired data from the buildings.

\section{Results and Discussion}

Firstly, both apartment buildings were modelled applying the indoor temperature model (Hietaharju et al., unpublished). Modelling results are presented in Table 2. The model performance was evaluated by calculating mean absolute error (MAE), mean absolute percentage error (MAPE), and root mean squared error (RMSE).

Table 2. Performance of the Indoor Temperature Model.

\begin{tabular}{lll}
\hline & Building 1 & Building 2 \\
\hline MAE $\left({ }^{\circ} \mathrm{C}\right)$ & 0.24 & 0.38 \\
MAPE $(\%)$ & 1.07 & 1.69 \\
RMSE $\left({ }^{\circ} \mathrm{C}\right)$ & 0.33 & 0.44 \\
\hline
\end{tabular}




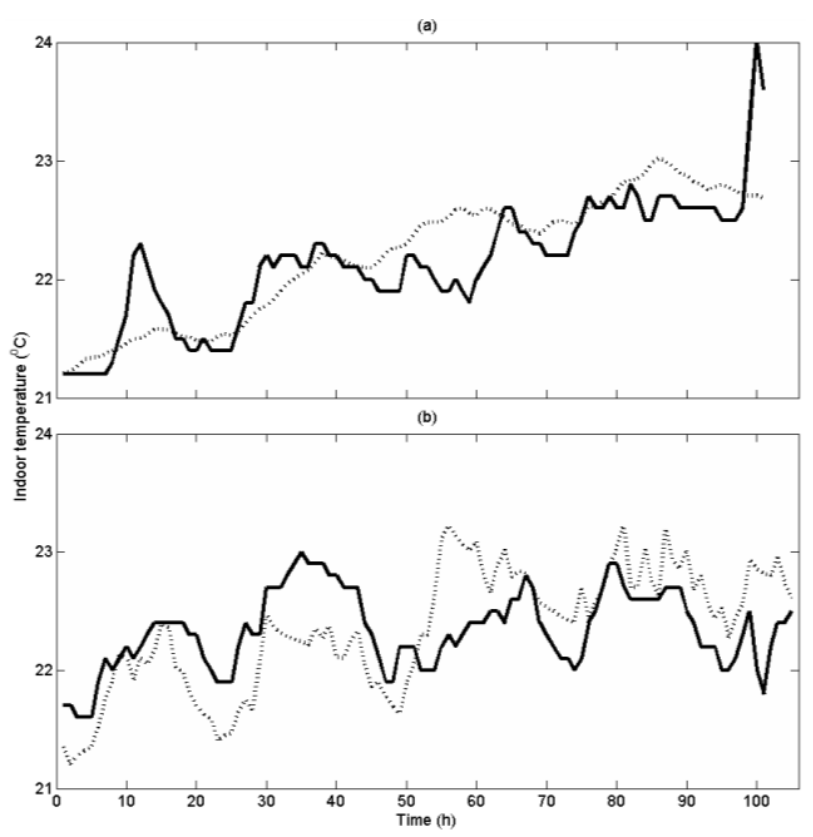

Figure 1. Measured (solid line) and predicted (dotted line) indoor temperature: (a) Building 1 and (b) Building 2.

Figure 1 shows the measured and the modelled indoor temperatures with data from Building 1 and Building 2 . The time period for simulations was from January 3rd 2015 to January 7th 2015. For the Building 1, indoor temperature measured from the living room was used as a reference. Respectively, for the Building 2 the temperature measured at the hallway was the model reference. It is important to notice the dynamic behavior that the model manages to capture. Although the modelled indoor temperature somewhat deviates from the measured temperature, the changes and trends are still captured by the model. This model property is further emphasized if the model is to be used for control purposes. Also, MAE, MAPE, and RMSE seem to be reasonably low in case of the two building data sets.

Next, different peak load cutting scenarios were simulated utilizing the identified indoor temperature models. For both of the buildings $30 \%, 50 \%$, and $70 \%$ peak load cuts during morning hours between 7 and 10 am were applied in the simulation. Value of $22{ }^{\circ} \mathrm{C}$ was assumed to be the minimum desired indoor temperature for both of the buildings during the simulation. The upper limit for the indoor temperature was set to $23{ }^{\circ} \mathrm{C}$ for the Building 2 and to $24^{\circ} \mathrm{C}$ for the Building 1 . These upper limits were determined from the historical indoor temperature data. Figure 2 and Figure 3 show the simulation results for the peak load cutting. For the indoor temperature, measured and optimized indoor temperatures are presented. The grey bar in the district heating graph represents the peak load period during which the maximum allowed heating power was restricted. Restricted power values were calculated from the measured district heating power by taking the average of the measured district heating power during the peak load period and multiplying this by the desired cut percentage.

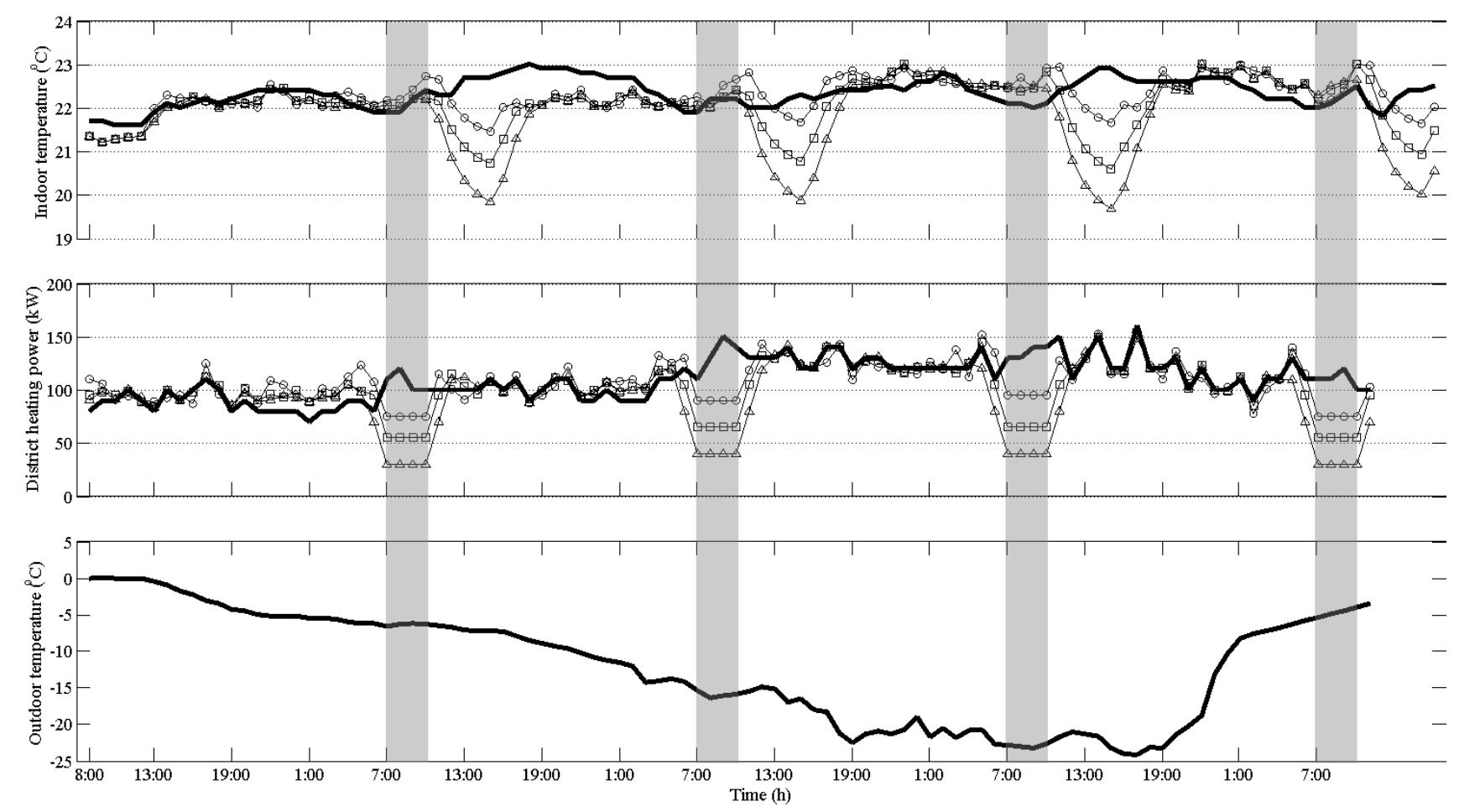

Figure 2. Simulation results for Building 2 in the case of 30\%, 50\%, and 70\% peak load cut during 7-10 am (grey bars). Upper: measured (solid line) and simulated (30\%: circle; 50\%: square; 70\%: triangle) indoor temperature. Middle: measured (solid line) and simulated (30\%: circle; 50\%: square; 70\%: triangle) district heating power. Lower: measured outdoor temperature. 
In Figure 2 for the Building 2, a clear change in indoor temperature can be seen as a result of peak load cutting. Delay in the indoor temperature response is due to the lag in the applied model. For the $30 \%$ peak load cut it can be seen that at first, the temperature rises to the set upper limit as heat is stored into the building before the peak load period. Then, the temperature drops as the heating power is limited to lower level during the peak loads. In the case of Building 2, the indoor temperature drops below the minimum of $22{ }^{\circ} \mathrm{C}$ in all of the simulated peak load cutting scenarios. For $50 \%$ and $70 \%$ peak load cuts the storage capacity of the building is not enough and the indoor temperature decreases significantly due to the peak load cutting. For the simulation period, the total energy savings are $2.2 \%$, $7.5 \%$, and $15.8 \%$ for the $30 \%, 50 \%$, and $70 \%$ peak load cuts respectively.

As can be seen in Figure 3, the simulated change in indoor temperature in the Building 1 is small when compared with simulation results in case of the Building 2 staying between 22 and $23{ }^{\circ} \mathrm{C}$. Nevertheless, the increase in temperature can be observed before the peak load period. Also, it can be clearly seen that the indoor temperature is higher in general on larger peak cuts to be able to perform the peak cut without compromising with the indoor temperature. Unlike in Building 2, the indoor temperature remains above $22{ }^{\circ} \mathrm{C}$ in Building 1 . Total energy savings for the $50 \%$ and $30 \%$ peak load cuts for the simulation period were $1.0 \%$ and $2.9 \%$ respectively. With 70\% peak cut energy is not saved during the simulation period but $2.3 \%$ more is needed. These results are much lower in comparison with the savings achieved for Building 2 . This is partly caused by the high peak in the first four hours in the heating power, which can be seen in Figure 3. This is due to the fact that the initial measured indoor temperature in Building 1 is below the desired level and it has to be raised. If the heating power for these first four hours is excluded from the total energy saving calculations, total energy savings are $6.2 \%, 4.1 \%$, and $0.7 \%$ for the $30 \%, 50 \%$, and $70 \%$ peak load cuts respectively. This shows that energy is saved with every peak load cut percentage, but the savings are still lower, except for $30 \%$ cut, when compared with Building 2. This results from the fact that in Building 2 heat is not significantly stored with $50 \%$ and $70 \%$ peak load cuts as the storage capacity is not enough to compensate for such large cuts and therefore the total energy savings are significantly higher in comparison with Building 1 but the indoor temperature has to be compromised. Higher savings for the $30 \%$ peak load cut in Building 1 compared with Building 2 can be explained with larger heat storage capacity in Building 1. In Building 2, 30\% peak load cut requires all heat storage capacity to be utilized as can be seen in Figure 2 where the indoor temperature rises to the maximum limit of $23{ }^{\circ} \mathrm{C}$ before the peak load cut periods. In Building 1 , heating power can be kept on a

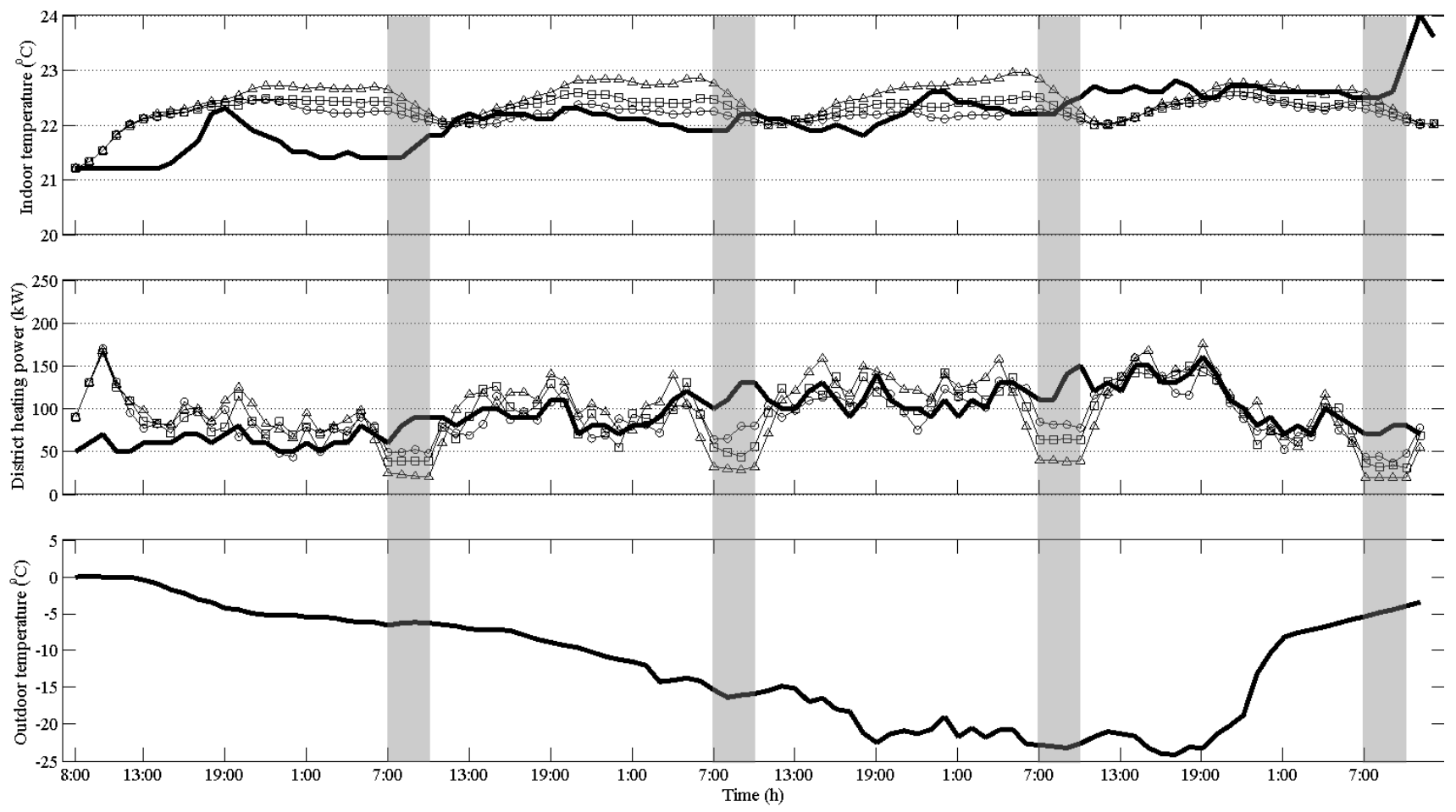

Figure 3. Simulation results for Building 1 in the case of 30\%, 50\%, and 70\% peak load cut during 7-10 am (grey bars). Upper: measured (solid line) and simulated (30\%: circle; 50\%: square; $70 \%$ : triangle) indoor temperature. Middle: measured (solid line) and simulated (30\%: circle; 50\%: square; 70\%: triangle) district heating power. Lower: measured outdoor temperature. 
lower level as the heat storage capacity of the building is more than enough to manage $30 \%$ cut in peak loads.

It can be seen from the results that the two buildings have different heat storing capacities. In this view, the Building 2 lacks the heat storing capacity to maintain the desired indoor temperature during the peak load cut of $30 \%$ or more, which can be clearly seen in Figure 2. However, the indoor temperature could be allowed to drop below the desired level for the duration of the peak loads. In the case of $30 \%$ peak load cut, the indoor temperature in Building 2 decreases at most about 0.5 ${ }^{\circ} \mathrm{C}$ below the desired temperature, which could be very well allowed. With $50 \%$ peak load cut the indoor temperature drops $1{ }^{\circ} \mathrm{C}$ below the desired temperature, which could still be allowed. If temperature drops of the same kind were also allowed for Building 1, the total energy savings would be more significant. On the other hand, also the maximum indoor temperature could be allowed to rise higher to get more heat storage capacity and ensure that indoor temperature does not drop below the desired level, but this could raise the total energy consumption. This shows how important it is to properly define the constraints for the optimization. Also, it is worth noticing that the studied buildings are apartment buildings where the indoor temperature must be more strictly maintained at acceptable level than for example in a school or an office building where the indoor temperature can be allowed to fluctuate more freely during the off-hours. In these kind of buildings, the use of building thermal mass could be even more effective. This is further supported by the preliminary results from an online test performed by the authors in a school building (Hietaharju and Ruusunen, 2015). There the district heating power was optimized for a 24-hour period and it resulted in 14\% energy savings for the period and an average of $25 \%$ peak load cut during the morning hours. Nevertheless, buildings exist with different heat storing capacities and therefore it is important to investigate multiple buildings as the city level peak loads are desired to be cut. In that case, the peak cutting would be distributed between the buildings and their storage capacities could be effectively used without extensively variating the indoor temperature of individual buildings. Systems with groups of buildings have already been investigated but will be further studied in the future.

\section{Conclusions}

An indoor temperature model was applied to simulate different peak load cutting scenarios of district heating. According to the simulations, peak cutting potential in the tested two buildings varied because of different heat storing capacities. Nevertheless, in both of the buildings peak loads could be cut $30 \%, 50 \%$, and even $70 \%$. In the Building 1, performing the peak cutting did not have a significant effect on the indoor temperature and it stayed between the desired levels. In the Building 2, the heat storing capacity was not enough to cut the peak loads by $30 \%$ or more without the indoor temperature fluctuating out of the defined limits. This shows the importance of properly defined constraints for the indoor temperature. Furthermore, these simulations demonstrate that the buildings have different heat storing capacities and therefore it is important to investigate systems with multiple buildings. In that case, the load cutting will be distributed between the buildings and their specific heat storing capacities can be effectively used to take into account variation in the temperature dynamics.

All the results presented in this article are simulations based on actual measured data. The next phase would be to conduct field-tests to evaluate the method's performance in a real building environment. In this article, peak load cutting and optimization is considered in a single building, but the overall goal is to develop optimization and peak load cutting methods for a system of multiple buildings. Having multiple buildings changes the picture completely and one can make conclusions about the energy savings also on city level. Systems with multiple buildings have already been investigated by the authors, but will be further studied in the future. Simulations presented in this article show that better energy efficiency in district heating can be achieved by predicting the energy consumption and utilizing the thermal mass of a building.

\section{Acknowledgement}

This work has been funded by the Finnish Funding Agency for Innovation (TEKES) as part of the KLEI project (40267/13). Authors thank Jyväskylän Energia Oy and Jyväskylän Vuokra-asunnot Oy for providing data for this study.

\section{References}

J. E. Braun. Load control using building thermal mass. Journal of Solar Energy Engineering, 125(3):292-301, 2003. doi:10.1115/1.1592184.

A. Eisentraut and A. Brown. Heating without Global Warming - Market Developments and Policy Considerations for Renewable Heat. IEA, Paris, France, 2014.

C.-E. Hagentoft and A. S. Kalagasidis. Effect Smart Solutions for District Heating Networks Based on Energy Storage in Buildings. Impact on Indoor Temperatures. Energy Procedia, 78:2244-2249, 2015. doi: 10.1016/j.egypro.2015.11.346.

G. P. Henze, T. H. Le, A. R. Florita, and C. Felsmann. Sensitivity Analysis of Optimal Building Thermal Mass Control. Journal of Solar Energy Engineering, 129(4):473485, 2006. doi: 10.1115/1.2770755.

P. Hietaharju and M. Ruusunen. A concept for cutting peak loads in district heating. In Proceedings of the Automaatio XXI seminar, Helsinki, Finland, 2015.

P. Hietaharju, M. Ruusunen, and K. Leiviskä. A parametric physical model for indoor temperature prediction and control in buildings. Unpublished. 
J. Kensby, A. Trüschel, and J.-O. Dalenbäck. Potential of residential buildings as thermal energy storage in district heating systems - Results from a pilot test. Applied Energy, 137:773-781, 2015. doi: 10.1016/j.apenergy.2014.07.026.

F. Ståhl. Influence of thermal mass on the heating and cooling demands of a building unit. $\mathrm{PhD}$ Thesis, Chalmers University of Technology, Sweden, 2009.

Y. Sun, S. Wang, F. Xiao, and D. Gao. Peak load shifting control using different cold thermal energy storage facilities in commercial buildings: A review. Energy Conversion and Management, 71:101-114, 2013. doi: 10.1016/j.enconman.2013.03.026. 